\title{
XII.
}

\section{Zur Frage der Schussverletzung des Magen-Darmtractus.}

\author{
You \\ Dr. med. Paul Klemm, \\ Assistenzarzt der chirurg. Abtheilung des Stadtkrankenhanses zu Riga.
}

Was thun wir nach perforirenden Schuss- oder Stichverletzungen des Bauches? Sollen wir abwarten oder operiren? Schon lange hat diese Frage das lebhafte Interesse der Kriegs- und Friedenschirurgen in Anspruch genommen, und mit Recht, denn Verletzungen dieser Art gehören auch in der Friedenspraxis nicht zu den Seltenheiten, und die Resultate der Behandlung sind zur Zeit noch so wenig erfreulich, dass es sich der Mühe verlohnt, zur Klärung dieser Dinge beizutragen.

Trotz der zahlreichen Debatten in chirurgischen Congressen und medicinischen Gesellschaften ist bis zur Stunde noch keine Einigung in der Frage der Behandlung perforirender Bauchschüsse erzielt worden. Zum Theil liegt der Grund dafür wohl auch darin, dass man, um einen Maassstab für die Leistungsfähigkeit der einen oder anderen Methode zu finden, einzig die Resultate der statistischen Ergebnisse beriicksichtigte. Man stellte einfach die exspectativ bestehenden Fälle zusammen, berechnete den Procentsatz der Mortalität und setzte denselben in Parallele mit dem der operativ behandelten Kranken. Dass ein derartiges Verfabren nicht zum Ziele führen konnte, lag auf der Hand.

Vergegenwärtigen wir uns nur einmal die Menge der Fehlerquellen!

Die neveste und genaueste Statistik stammt von Paul Reclus.)

Er verwerthete sein Material in der Weise, dass er auf der einen Seite die operativ behandelten Fälle zusammenstellte, geordnet-nach der zwischen der Verletzung und der darauf folgenden Laparotomie verstrichenen Zeit; auf der anderen Seite führt er die exspectativ

1) Reclus et Noguès, Traitement des perforations traumatiques de l'estomac et de l'intestin. Revue de Chirurgie. 1890. 2 u. 5. 
behandelten Fälle an. Die Resultate beider Reihen verglich er mit einander.

Sehen wir ganz ab von der Schwierigkeit der Diagnose einer perforirenden Schussverletzung des Bauches mit Betheiligung der Eingeweide, so muss man jedenfalls verlangen, dass in einer Statistik, deren Resultate entscheidenden Werth beanspruchen, noch andere Punkte berticksichtigt werden, als allein die Länge der zwischen Verletzung und Operation verstrichenen Zeit.

So ist doch z. B. der von der Läsion betroffene Abschnitt des Magen-Darmtractus in Rechnung zu ziehen, da die verschiedene Werthigkeit der Wunden des Dünndarms gegenüber denen des Magens und Dickdarms. bekannt ist. Beachtenswerth ist ferner die Menge und Grösse der Perforationsöffnungen, ihre Lage zu einander und zum Darm. Je grösser die Darmwunde, desto reichlicher kann Koth austreten, und je näher zum Mesenterialrand des Darmes sie gelegen ist, desto eher tritt die Gefahr der Darmgangrän an das Eingeweide heran.

Lassen wir auch die individuell ja verschiedene Dexterität und Erfahrung des einzelnen Operateurs auf dem Gebiet der Bauchchirurgie ausser Acht, so muss doch auf die Methode der operativen Therapie im einzelnen Falle eingegangen werden. Wir wissen, dass es sich nicht gleich bleibt, ob wir nach einer Laparotomie die Peritonealhöhle ausspülen, mit trockener Gaze austupfen, ob wir sie tamponiren, drainiren oder die Bauchhöhle durch die Naht völlig schliessen.

Von diesen Dingen findet sich in den bekannten Statistiken kein Wort, und doch findet gerade auf diesem schwierigen Gebiet das Wort, dass wir unsere Erfolge oder Misserfolge nicht zählen, sondern wägen sollen, seine vollste Berechtigung.

Die pathologisch - anatomische Würdigung des einzelnen Falles ist es, die zu richtigeren Anschaungen geftihrt hätte, als die statistische Verwerthung des Materials. Das Studium des Mechanismus der perforirenden Bauchverletzungen, sowie die Erkenntniss der pathologischen Processe, die sich an dieselben schliessen, sind die Factoren, die eine richtige Basis für die einschlägige Therapie bilden sollten.

Ich habe nun an der Hand des Thierexperiments versucht, die Anatomie und Pathologie der Schussverletzungen des Unterleibes, speciell des Magen-Darmkanals näher kennen zu lernen, und möchte mir erlauben, indem ich mir eine eingehendere Darlegung dieses Gegenstandes vorbehalte, an dieser Stelle einige kurze Bemerkungen uber die Folgen der traumatischen Magen-Darmperforation zu machen. 
Wegner's') classische Arbeit beleuchtete zum ersten Mal in klarer, exacter Weise die physiologischen und pathologischen Vorgänge am Bauchfell.

Nach seiner Auffassung stellt die Peritonealhöhle einen grossen Binnenraum des Bindegewebes dar, an dem uns folgende Eigenschaften besonders interessiren:

1. die bedeutende Flächenausdehnung;

2. die enorme Resorptionsfähigkeit;

3. die ebenso grosse Transsudationsfähigkeit;

4. die Plasticität des dieselbe einschliessenden Gewebes.

Von der grössten Wichtigkeit ist der zweite Punkt. Wie Wegner an einer grossen Zahl lehrreicher Experimente zeigte, ist die bedeutende Resorptionskraft des Bauchfells der beste Schutz desselben gegen eingedrungene Schädlichkeiten. Er konnte fäulnissfähige Substanzen, wie Muskelinfus, Serum, Milch mit Luft u. s. w., in die Bauchhöhle injiciren, ohne dass Peritonitis eintrat. Die eingebrachten Körper wurden schnell aufgesogen und in das circulirende Blut aufgenommen, wo sie unschädlich gemacht wurden. Erst dann, wenn die Menge der injicirten Substanz in einem Missverhältniss zur Resorptionskraft des Peritoneums stand, so dass nur ein Theil derselben resorbirt wurde, während der andere stagnirte und der Zersetzung durch die eingedrungenen Mikroorganismen anheimfiel, fand eine Infection des Gesammtorganismus statt. Hier gereichte die resorbirende Kraft des Peritoneums dem Körper zum Schaden. Sie bewirkte eine Ueberschwemmung der Gesammtblutmasse mit Mikroben, sowie deren Stoffwechselproducten, der der Körper unter dem Bilde der acuten oder mehr chronischen Intoxication unterlag. Nach Wegner ist es mithin nicht die Peritonitis, d. h. die Gewebserkrankung des Bauchfells im pathologisch-anatomischen Sinne, die den Tod des Patienten herbeiführt. Nach seiner Ueberzeugung stellt das Peritoneum nur die Eingangspforte dar, durch welche die giftigen Substanzen in den Säftekreislauf aufgenommen werden. Er präcisirte seinen Standpunkt in folgenden Worten: „Will man die wahre causa mortis bezeichnen, so sollte mannicht, wiedieses gewöhnlich geschieht, die Peritonitis anschuldigen, sondern die durch die Resorption herbeigefuhrte Blutvergiftung im chemischen und corpusculären Sinnedieses Wortes".

1) Chirurgisches über die Peritonealhöhle, mit besonderer Berücksichtigung der Ovariotomie. Langenbeck's Archiv. Bd. XX. 
Von eminenter Wichtigkeit tritt uns hier zum ersten Male die. Thatsache entgegen, dass das Bauchfell, d. b. wohlverstanden das gesunde, mit normaler Resorptionskraft ausgestattete Peritoneum, eine gewisse Menge fäulnissfähiger, ja selbst toxischer Substanzen ohne Schaden, sowobl für sich, als auch den Gesammtorganismus, zu resorbiren vermag.

In neuester Zeit bat Reichel') in Wtirzburg, gestitzt auf die von Wegner gefundenen Thatsachen, einen sehr werthvollen Beitrag zur Aetiologie der septischen Peritonitis geliefert.

Er injicirte Thieren eine gewisse Menge Sepsis-, sowie Eiter erregender Spaltpilze in die Bauchböhle, ohne dass dieselben in ihrer Gesundheit beeinträchtigt wurden; ebenso konnte er bestimmte Mengen flüssigen Kothes einbringen, obne dass Peritonitis eintrat. Erst bei Injection reichlicherer Mengen gingen die Thiere unter den Erscheinungen der schweren septischen Intoxication zu Grunde. Zur Bildung einer diffusen, exsudativen Peritonitis kam es in der Regel nicht.

Halten wir die von Wegner und Reichel in einer grossen Zahl einwurfsfreier Experimente gefundenen Thatsachen fest und betrachten wir von diesem Standpunkt aus die Verletzungen des MagenDarmtractus!

Diese geben uns ein völlig reines Analogon zu den Thierexperimenten der beiden genannten Autoren. Sowohl im Versuch, als auch bei dem den Magen oder Darm treffenden Trauma kommt es zum Eintritt septischer Substanzen in die normale Bauchböble. Von dem Kotherguss, wie er nach Perforation infolge geschwüriger Processe intraabdominal gelegener Hohlorgane auftritt, müssen wir natürlich absehen, da hier die physiologischen Eigenschaften des Bauchfells durch die vorhergegangenen entzündlichen Processe wohl stets tangirt sein werden und die Verhältnisse für eine schnelle Resorption der eingedrungenen Schädlichkeiten hier ungünstig liegen.

Nehmen wir den Fall, es habe eine perforirende Schussverletzung des Abdomens mit Durchbohrung des Darms stattgefunden; wie gestalten sich die pathologischen Processe in der weiteren Folge?

Theoretisch lassen sich mehrere Möglichkeiten construiren: die Kugel hat den Darm perforirt, es ist Koth ansgetreten; findet nun ein spontaner Verschluss der Perforationsöffnung statt, so kann die ins Peritonealcavum gedrungene Kothmenge resorbirt werden und der Patient genesen, ja es braucht nicht einmal zum Erguss von

1) Beiträge zur Aetiologie und chirurg. Therapie der septischen Peritonitis. Deutsche Zeitschrift für Chirurgie. Bd. XXX. Heft 1. 
Koth zu kommen, da der Verschluss der Wunde so schnell erfolgen kann, dass Kothaustritt verhindert wird. Andererseits lässt sich aber der Fall so formuliren, dass ein Verschluss der Perforationsöffnung im lädirten Eingeweide nicht so bald oder überhaupt nicht ausgiebig genug stattfindet, sondern dass während eines gewissen Zeitraumes in bestimmten Intervallen Koth in die Bauchhöhle fliesst, somit auch längere Zeit hindurch eine Resorption septischer Substanzen stattfindet.

Die beiden hier kurz skizzirten Vorstellungen finden ihre Vertheidiger im Lager Derer, die zunächst bei der tablichen Medication warten wollen, bis sich die Symptome der Peritonitis einstellen, und erst dann einen operativen Eingriff in Erwägung ziehen, und in dem der "Interventionisten ", die in jedem Fall einer perforirenden Verletzung des Abdomens die Laparotomie zur Aufsuchung und Naht des verletzten Eingeweides empfehlen.

Die Parteigänger letzterer Richtung finden sich besonders zahlreich in Amerika und England, während in Frankreich hauptsäcblich der exspectativen Behandlung gehuldigt wird.

Ihren Hauptvertreter findet dieselbe in Reclus, dessen Glaubens- und Lehrdogma etwa folgendermaassen lautet: Eine grosse Zabl von perforirenden Verletzungen des Abdomens mit Betheiligung des Magens und Darms durch Schuss und Stich kann spontan ausheilen. Es geschieht das in der Weise, dass die Oeffnung in der Darmwand durch einen in dieselbe prolabirenden Ptropf der Schleimhaut verschlossen wird, wie das auch schon vor ihm viele Autoren gelehrt haben. Dieser Schleimhautverschluss ist zunächst nur ein provisorischer; indem sich in der Umgebung der Perforationsöffnung feste Verwachsungen mit benachbarten Schlingen der vorderen Bauchwand oder dem Netz bilden, wird derselbe zu einem definitiven. Theoretisch lässt sich diese von Reclus und seiner Schule ins Feld geftihrte Deduction ja wohl halten, doch steht sie leider im Widerspruch zu den Angaben der meisten Autoren, die viel Magen- und Darmverletzungen zu sehen Gelegenheit hatten und spontane Ausheilung derselben nur in den seltensten Fällen beobachteten.

Um die Frage von der pathologisch-anatomischen Seite zu fassen und erst einmal zuzusehen, wie denn die Schussverletzungen des Magens und Darms eigentlich aussehen, experimentirte ich in der Weise, dass ich auf Hunde, Katzen und Kaninchen Schüsse abgab. Die auf ein Brett gebundenen Thiere wurden aufrecht an die Wand gestellt. Der Schuss erfolgte aus einer Distanz von 7-8 Schritt mit einem Projectil von $5 \mathrm{Mm}$. Durchmesser. Ich benutzte sowohl 
einen Revolver, als auch eine sogenannte Montechristo-Pistole, sowie Spitz- und Rundkugeln.

Folgende Punkte waren es, auf die ich zunächst meine Aufmerkkeit richtete:

1. die Grösse der Wunde;

2. das Aussehen und die Beschaffenheit derselben;

3. die Multiplicität.

Hinsichtlich des ersten Punktes kann ich der Anschauung vieler Autoren, dass zwisehen der Grösse des abgeschossenen Projectils und der durch dasselbe verursachten Perforation der Darmresp. Magenwand ein bestimmtes Verhältniss existire, nicht beipllichten. Ich war vielmehr uberraseht durch die grosse Mannigfaltigkeit in der Grösse der Wunden. Es blieb sich dabei gleich, ob ich den Montechristo oder den Revolver, ob ich Rund-oder Spitzkugeln benutzte. Das eine Mal hatte ich bei grossen Hunden einen Defect von 3-4 $\mathrm{Cm}$. Durchmesser, während ich das andere Mal unter den gleichen Bedingungen Perforationen erzielte, deren Durchmesser dem des gebrauchten Projectils gleichkam. Es erklärt sich diese Verschiedenheit in der Grösse der Darmwunden aus der Grösse des Winkels, unter dem die Kugel das Eingeweide berthhrt. Trifft dieselbe die Darmwand senkrecht, so entsteben Verletzungen, deren Durchmesser dem des gebrauchten Geschosses annäbernd adäquat ist. Wird der Winkel kleiner als ein rechter, so gewinnt die Wunde mehr den Typus der Längs- oder Querwunde des Darms, je nachdem das Projectil parallel zur längsaxe desselben oder senkrecht zu dieser aufgesetzt wurde. Je näher zur Peripherie des Darmrohres die Kugel traf, desto grösser waren die resultirenden Defecte; sie betrafen gelegentlich die halbe Darmwand, wie ich bei meinen Versuchsthieren dieses mehrere Male beobachten konnte. Traf die Kugel so, dass sie die Darmwand nur in einem Punkte berubrte, so resultirten daraus Verletzungen, die nur die Serosa oder Serosa und Muscularis betrafen - reine Tangentialschüsse.

Es ist interessant, hierïber die Angaben von $\mathrm{Bogdanik}^{1}$ ) zu vergleichen, der gelegentlich der Volksunruhen in Biala, die ein Einschreiten der bewaffneten Macht nöthig machten, Gelegenbeit hatte, eine Reihe von Schussverletaungen des Darms zu sehen, die durch Projectile des Mannlicher-Gewehres verursacht worden waren. Dieselben besitzen ein Kaliber von $8 \mathrm{Mm}$. mit einer Anfangsgeschwindigkeit von 530 Meter. Der Durchmesser der Darmwunden war

1) Die Geschosswirkung der Mannlicher-Gewehre. Wien. Klinik. 1890. Heft 12. 
trotz der eminenten Durchschlagskraft der Projectile meist grösser, als der letzterer selbst. Wir finden unter den gesammelten Fällen Verletzungen des Darms von 1, 2 und $3 \mathrm{Cm}$. Ausdehnung. Diese Thatsachen widersprechen jedenfalls der von Reclus vertretenen Ansicht, wenn er in seiner schon vorhin citirten Arbeit in der Revue de Chirurgie Nr. 2. 1891. p. 91 sagt: „Nous n'ignorons pas que s'il prend l'intestin en écharpe, un petit projectil peut provoquer des déchirures étendues et bien supérieures à son diamètre, mais ces cas sont rares et nul ne le conteste, on pouvait comparer, toutes choses égales d'ailleurs, les lésions imputables à un revolver de poche ou une carabine de salon aux désordres, produits par un fusil de guerre ou un éclat d'obus."

Jetzt noch einige Worte iber das Ausseben der Wunden!

Der Charakter war immer der gleiche; ganz einerlei, ob es sich um kleinere oder grössere Defecte handelte, bot die Wunde stets die Zeichen stattgehabter Quetschung dar. Die Ränder waren unregelmässig gerissen und gefetzt, die nächste Umgebung zeigte Suffusionen und Ekchymosen; am prononcirtesten trat das an der Schleimbaut hervor, die im Umkreise mehrerer Millimeter blutig infiltrirt war. Aus der Anatomie dieser Wunden geht schon hervor, ob ein spontaner Verschluss derselben durch Schleimhautprolaps erfolgen, resp. eine genligende Garantie für seine Schlussfähigkeit bieten kann. In jedem meiner Experimente konnte ich constatiren, dass die gefetzte Mucosa in den Defect der Darmwand prolabirt war. Dieses Verhalten ist ja auch natürlich, wenn man in Rechnung zieht, dass die Scbleimhaut massiger und voluminöser ist, als die übrigen Strata der Darmwand, da sie bei gleicher Ausdehnung mehr Oberfläche besitzt, als Serosa und Muscularis. Ferner uberzeugte ich mich aber auch, dass auch in Fällen, wo der Durchmesser der Darmwunde $7-8 \mathrm{Mm}$. nicht tiberstieg, es sich also um relativ kleine Oeffnungen handelte, ein Verschluss derselben durch vorgestiulpte Schleimbaut nicht erfolgt war. Letztere war hier und da locker mit dem Rande der Perforationsöffnung verklebt, aber eine sichere, das Austreten von Koth verhindernde Obturation war kein einziges Mal erfolgt.

Da diese Frage doch von beträchtlicher Wichtigkeit und Tragweite ist, weil ibre Entscheidung uns allein in der Wahl der einschlägigen Therapie zu leiten vermag, untersuchte ich weiter, ob tiberhaupt und unter welchen Verbältnissen ein spontaner Verschluss einer Magenresp. Darmwunde durch einen Schleimhautpfropf entstehen könne.

Bei frisch getödteten Thieren etablirte ich Stichwunden des Darms mit einem scharf geschliffenen, spitzen Scalpell, die Wunden, 
ca. 4-5 Cm. lang, durchsetzten die ganze Dicke der Darmwand und waren absolut glattrandig. Sofort nach dem Herausziehen des Messers stülpte sich Schleimhaut vor und füllte wie ein festsitzender Pfropf das ganze Lumen der Darmwunde aus, so dass man fraglos den Eindruck gewinnen musste, als ob ein derartiger Verschluss für die absolute Obturation einer Magen- oder Darmwunde völlig gentigte. Nach 1-2 Minuten Abwarten, bisweilen etwas früher oder später, änderte sich das Bild. Im Abschnitt oberbalb der Darmwunde ging die Peristaltik ruhig weiter; unter dem Druck der bewegenden, peristaltischen Welle liess sich nun verfolgen, wie der Pfropf immer tiefer ins Lumen der Darmwunde getrieben wurde, bis auf der Kuppe desselben ein Tröpfehen Koth erschien. Dieses Spiel wiederholte sich mehrere Male, bis es mit dem Erlöschen der Peristaltik aufhörte. Es gelangte also während eines gewissen Zeitraumes eine bestimmte Kothmenge ins Cavum peritoneale. Und in der That konnte ich bei Thieren, die ich 5 Minuten nach experimentell erzeugtem Bauchschuss tödtete, stets freien Koth in der Bauchhöhle nachweisen.

Der oben geschilderte Befund macht die Annahme vieler Autoren, dass der erwähnte Schleimhautpfropf einen gentigend sicheren Verschluss einer Magen- oder Darmwunde bilden könne, verständlich. An der Leiche, wo die treibende Kraft der Peristaltik fehlt, muss man fraglos die Ueberzeugung gewinnen, als ob die Wunde sicher geschlossen sei, zumal man in der Regel keinen freien Koth findet, was ja nicht befremden darf, da derselbe zum Theil resorbirt, zum Theil in dem ergossenen Blute aufgelöst ist.

Was den dritten Punkt, die Multiplieität der Darmwunden, anbetrifft, so befinde ich mich in vollster Uebereinstimmung mit Denen, die solitäre Verletzungen des Magen-Darmkanals durch Schusswaffen zu den Seltenheiten zählen.

Aus einer Zahl von 32 Versuchen ergab sich im Durchschnitt eine dreifache Perforation pro Thier. Das Maximum war eine neunfache Durchlöcherung des Darmes, einmal hatte die Kugel die Bauchhöhle durchsetzt, obne Magen oder Darm verletzt zu haben, es war nur eine Läsion der Milz mit beträchtlichem intraperitonealem Bluterguss zu Stande gekommen. Eines der Thiere erholte sich nach einer offenbar perforirenden Schussverletzung des Bauches vollkommen, doch bei der Autopsie stellte es sich heraus, dass die Kugel die Peritonealhöhle nicht durchsetzt, sondern das Bauchfell nur gestreift hatte.

Gerade zu der Zeit, als ich mit obigen Versuchen beschäftigt 
war, hatte ich Gelegenheit, einen Fall von Schussverletzung des Abdomens zu sehen und den Befund mit dem an meinen Versuchsthieren zu vergleichen. Derselbe stammte aus der Praxis des Herrn Docenten Dr. von Zöge-Mantenffel und danke ich ihm die freundliche Ueberlassung desselben.

Adolph Thomassohn, 6 Jahre alt, aus Dorpat, hatte sich am 9. Juli 189110 Uhr Morgens beim Spielen mit einem Revolver in den Leib geschossen. Das Projectil hatte ein Kaliber von $5 \mathrm{Mm}$. - Gleich nach der Verletzung soll Erbrechen stattgefunden haben, doch soll kein Blut im Erbrochenen gewesen sein.

Status praes.: Patient ist mittelgross, normal entwickelt, gut genährt. Allgemeine hochgradige Anämie. Die Athmung ist oberflächlich und frequent. Puls 100, sehr klein. Das Abdomen ist in toto stark aufgetrieben, die Leber nach oben verdrängt, gegen Berihrung sehr em. pindlich.

Im Epigastrium, 4 Finger breit unter dem Rippenbogen, dicht über dem Nabel, $2 \mathrm{Cm}$. nach links von der Mittellinie eine $6 \mathrm{Mm}$. im Durchmesser haltende Einschussöffnung; die Ränder derselben sind gequetscht, die Umgebung von Pulverschmauch geschwärzt. Bei der 4 Stunden nach der Verletzung von v. Zoege-Manteuffel vorgenommenen Operation fand sich Folgendes: In der Chloroformnarkose wurde der Leib durch einen vom Proc. xyphoid. bis zur Symphyse reichenden Schnitt eröffnet. Aus der Bauchhöhle quoll fltusssiges Blut; es drängte sich eine zerschossene Darmschlinge vor. Nach Auspackung der Därme findet sich am Darm. kanal, der vom Magen ab successive abgesucht wurde, folgender Befund: Auf einer $80 \mathrm{Cm}$. langen Strecke des oberen Ileum vertheilt, lassen sich 11 Perforationsöffungen constatiren, deren Durchmesserverschieden gross sind; wir fanden neben solchen von 7-9 $\mathrm{Mm}$. auch solche, deren Durchmesser $1 \mathrm{Cm}$. bi s $1,5 \mathrm{Cm}$. betrug. Das Quercolon war in tangentialer Richtung angeschossen worden, so dass dasselbe einen $9 \mathrm{Mm}$. messenden Längsriss aufwies. Das Mesenterium der Flexura sigmoidea war durchbohrt.

Sämmtliche Darmwunden boten die Zeichen starker Quetschung; in der Umgebung derselben zeigten sich Sugillationen und feinste Hämorrbagien, besonders deutlich auf der Schleimlaut. Letztere hing in Fetzen in die Perforationsöffnungen der Darmwand hinein, von einem Ver sehluss derselben durch Pfropfbildung war nirgendwo etwas zu sehen, kurz der pathologisch-anatomische Befund in vorliegendem Falle war genauderselbe, wie bei meinen experimentell erzeugten Bauchschüssen. Die Bauchhöhle war mit dunklem, flïssigem Blut gefïllt, freier Koth war in derselben nicht zu bemerken.

Der Colonriss wurde angefrischt und darch die doppelreihige Lembert-Czerny'sche Naht geschlossen. Die ganze $80 \mathrm{Cm}$. lange Partie des Ileum wurde resecirt, nachdem die Bauchhöhle provisorisch durch einige Nähte geschlossen worden war, so dass nur die zerschossene Darmschlinge aussen lag. Das Mesenterium wurde in Portionen unterbunden; 
ein Keil worde aus demselben nicht geschnitten. Die quer durchtrennten Lumina des Darmes wurden durch doppelreihige Knopfnaht mit feinster Seide vereinigt. Nachdem die Nahtlinie jodoformirt war, wurde die Schlinge versenkt, die Bauchhöhle mit trockener Gaze ausgetupft und durch die Naht geschlossen. Die Dauer der Operation betrug $13 / 4$ Stunden; am Abend desselben Tages erfolgte bei dem stark geschwächten Patienten der Tod im Collaps.

Ich glaube aus meinen Versuchen zunächst Folgendes schliessen zu durfen:

1. Nach Sebuss- und Stichverletzungen des Magens und Darms findet ein Verschluss der Perforationsöffnungen durch einen Schleimhautpfropf, der dem Kotherguss in die Bauchhöhle vorbeugen könnte, nichtstatt; es tritt vielmehr stets Koth durch, und zwar hängt dieses ab:

a) von der Grösse und Beschaffenheit der Perforationsöffnungen; je grösser der Defect ist, je gefetzter und unregelmässiger die Ränder desselben sind, desto mehr Koth kann natürlich durchtreten;

b) vonder Menge der Perforationen.

Vergleichen wir jetzt noch einmal die Verhältnisse, wie sie nach perforirenden Bauchschüssen und -stichen liegen, mit denen, wie sie sich nach Injection flüssiger Mengen von Koth in die Peritonealhöhle von aussen her gestalten. In letzterem Falle wird der Organismus eben mit den eingedrungenen Schädlichkeiten fertig, oder er unterliegt; das hängt von den disponiblen reactiven Kräften desselben, sowie der eingebrachten Menge von Koth ab. Anders bei unseren Versuchen: hier tritt von Zeit zu Zeit immer wieder aufs Neue toxische Substanz in den Säftekreislauf über, die schliesslich eine Erschöpfung der reparativen Kräfte des Organismus, speciell des lebenden Blutes zu Wege bringt. Ferner ist hierbei noch eines weiteren Factors Erwähnung zu thun: der stets im Gefolge perforirender Bauchverletzungen eintretenden intraperitonealen Blutung. Dass reines Blut vom Bauchfell obne Schaden resorbirt werden kann, wissen wir; hier aber tritt eine Infection des Blutergusses durch den in denselben gelangenden Koth ein. Die Zersetzung desselben und damit die Etablirung eines intraperitoneal gelegenen Jaucheherdes kann die Folge sein, von dem aus dem circulirenden Blute beständig giftige Substanzen zuströmen.

Wie gestalten sich die pathologischen Processe am Thier, welches eine perforirende Bauchverletzung erlitten hat, nun weiter? Voraus- 
schicken will ich, dass alle Thiere bei exspectativer Behandlung starben.

Eine wahre Peritonitis, im Sinne der eitrigen Phlegmone des Bauchfells, konnte ich keinmal nachweisen; die Veränderungen am Peritoneum waren im Verhältniss zu den schweren Allgemeinerscheinungen sehr geringgradig.

Miculicz') unterscheidet nach Perforationen des Magens und Darms folgende Arten der Peritonitis:

1. Die peracute Form, die diffuse septische Peritonitis. Dieselbe fuhrt binnen Kurzem zum Tode und schliesst sich vorzüglich an Infectionen, die einen beträchtlichen Abschnitt des Bauchfells zu gleicher Zeit betroffen baben.

Es ist dieselbe Erkrankung, die König und Witzel als peritoneale Sepsis, Vercheré ${ }^{2}$ ) als Septicémie intestino-péritonéale beschrieben haben.

2. Die progredient fibrinös-eitrige Peritonitis.

Die Infection geht von einem kleinen, umschriebenen Bezirk aus. Es bilden sich anfangs Verklebungen, doch im weiteren Verlauf wird die schïtzende Barrière durchbrochen, der Process zieht immer nene Abschnitte des Bauchfelles in Mitleidenschaft, bis endlich in der Regel der Tod die Scene schliesst.

3. Die circumscripte eitrige Peritonitis, der intraperitoneale, abgekapseite Abscess.

Wie schon gesagt, habe ich keinmal freien Eiter in der Bauchhöhle gefunden; $a b$ der Grund in einer geringeren Neigung des thierischen Peritoneums zu eitrigen Processen, oder in völliger Abwesenheit der die Eiterung erregenden Mikroben zu suchen sei, kann ich nicht entscheiden. Es fehlten ferner auch in der Regel festere fibrinöse Verklebungen und Auflagerungen von Faserstoff. Ich kann mich in dieser Beziehung Reichel vollkommen anschliessen, der das nämliche Verhalten bei seinen Versuchsthieren constatirte.

Ich hatte bei meinen Experimenten nun hauptsächlich folgende pathologisch-anatomischen Befunde in der Bauchhöhle.

In einer Reihe von Fällen trat der Tod nach perforirenden Bauchschässen schon innerhalb weniger Stunden ein. Der Befund dabei ist wie in folgendem Versuch:

Am 27. März wurde ein kräftiger Kater um $10 \mathrm{Uhr}$ Morgens angeschossen. Einschuss in der Regio hypogastr. rechts von der Mittellinie. Nach 24 Stunden trat der Exitus ein.

1) Ueber Laparotomien bei Magen-Darmperforationen. Sammlung klin. Vortrăge. 1885 . Nr. 262 .

2) De la sépticémie intestino-péritonéale. Revue de Chirurg. 1888. p. 559. 
Section: In der Bauchhöhle kein Exsudat, kein fluissiges Blut, keine Coagula, keine fibrinösen Auflagerungen oder Verklebungen. Entsprechend der Einschussöffinung findet sich ein Dünndarmpacket durch ganz lockere Verklebungen der vorderen Bauchwand leicht adhärent. $5 \mathrm{Cm}$. oberhalb der Valv. Bauhini ist der Diunndarm doppelt perforirt, $10 \mathrm{Cm}$. höher abermalige doppelte Durchbohrung. Die Grösse der Oeffnungen schwankt zwischen $8 \mathrm{Mm}$. bis $1 \mathrm{Cm}$. Die Ränder sind gefetzt, gequetscht und sugillirt. Ein Schleimhautpfropf nirgends vorhanden. Das Peritoneum parietale et viscerale zeigt weder Infection der Gefässe, noch Trübung.

Einen ähnlichen Befund constatirte ich in einer ganzen Reihe von Versuchen sowohl an Katzen, als auch an Hunden, nur dass letztere viel toleranter gegen toxische Einwirkungen auf das Bauchfell sind, als erstere. Es ist das Bild der schweren, acut verlaufenden Vergiftung, bei mangelndem pathologiseh-anatomischem Befund, das diese Erkrankung charakterisirt. Der pathologische Process ist dabei offenbar der, dass ein ausgiebiger Kotherguss in die Bauchhöhle stattgefunden hat. Die Blutmasse ist durch Resorption rom Peritoneum her mit einer solchen Masse toxischer Substanzen tuberladen, dass ein Weiterbestehen des Lebens damit unvereinbar ist. Mikroskopirt man in solehen Fällen ein Tröpfehen Blut aus einem der Mesenterialgefässe oder auch der Vena jugul. comm., so ist man erstannt uber die zabllose Menge von Mikroben, die in demselben wimmeln.

In einer zweiten Gruppe von Fällen, die in etwas längerer Zeit - der Verlauf erstreckte sich oft uber einige Tage -- zum Tode führten, lagen die Verhältnisse wie etwa in folgendem Versuch:

Ein kräftiger, mittelgrosser Hund wird am 29. März angeschossen. Einschuss im Mesogastrium in der Mittellinie. Ausschuss nicht vorhanden. Nach 4 mal 24 Stunden erfolgte der Exitus.

Section: In der Bauchböhle eine beträchtliche Menge einer stark stinkenden, bräunlich-rothen Flüssigkeit. Peritoneum viscerale et parietale lebhaft injicirt und getrübt. Keine fibrinösen Beschläge. Der Darm ist hier und da lebhaft injicirt, die Schlingen sind eng, nicht mit einander verklebt. An der Vorderfläche des Magens befindet sich eine $3 / 4 \mathrm{Cm}$. im Durchmesser haltende Perforationsöffinung, aus welcher Schleimhaut prolabirt. Bei dem geringsten Zuge am Magen tritt durch die Oeffnung Mageninhalt aus, bis sich wieder einige Schleimhauttlocken vor die Oeffnung legen und den Austritt verhindern. An der Hinterfläche des Magens findet sich kein Ausschuss.

Hier ist der Vorgang ein etwas anderer: Zugleich mit der perforirenden Schussverletzung entstand ein intraperitoneales Hämatom, welches durch den austretenden Mageninhalt inficirt wurde. So 
etablirte sich ein Jaucheherd, von dem aus continuirlich dem Blute toxische Substanzen zuströmten.

Beide Gruppen von Fällen, sowohl die, wo bei der Autopsie sich in der Bauchböhle nichts nachweisen lässt, als auch die, welche sich durch Bildung eines intraperitonealen jauchigen Exsudates charakterisiren, gehören in die Kategorie der diffusen septischen Peritonitis von Miculicz oder der peritonealen Sepsis König's. Principiell unterscheiden sich beide Formen nicht wesentlich von einander. In der ersten Gruppe erfolgte der Tod schon durch Resorption des primären Kothergusses, in der zweiten war der Verlauf protrahïter: durch Zersetzung des in die Peritonealhöhle ergossenen Blutes fand eine Potenzirung der eingedrungenen Mikroben, sowie ibrer Stoffwechselproducte statt, deren continuirliche Resorption endlich die Wehrkraft des Organismus erschöpfte und den Exitus herbeiftuhrte.

Ich möchte an dieser Stelle auf die Analogie mit der eingeklemmten Hernia hinweisen: auch hier haben wir Fälle, wo trotz gelungenem Bruchschnitt und Reposition der eingeklemmt gewesenen Schlinge der Tod eintritt, ohne dass uns die Section einen befriedigenden Aufschluss über die Todesursache zu geben vermag. Es handelt sich auch in diesen Fällen um eine septische Infection des Organismus, die ihren Ausgang von der strangulirt gewesenen, in ihrer Ernährung beeinträchtigten Schlinge genommen hat. Den Beweis dafir baben wir in der Thatsache, dass in einer Reihe von Fällen nach Incarceration septisch-pnenmonische Herde, sowie Herde in der Leber und der Niere gefunden werden. Diese bezeichnen docb jedenfalls den Weg, den die vom Bauchfell resorbirten Substanzen im Körper zurluckgelegt haben. Immer ist es also die Beziehung der sich intraperitoneal abspielenden pathologischen Processe zum Gesammtorganismus, die wirim Auge behalten müssen. Von diesem Gesichtspunkt aus muss ich auch die von König gewählte Bezeichnung "peritoneale Sepsis" allen anderen vorziehen, weil sie eben das Punctum saliens, die vom Bauchfell ber inducirte Blutvergiftung am prägnantesten ausdrickt. Wir sehen ja oft grosse intraperitoneale Eiterherde, besonders nach perityphlitischen Processen, die, falls sie abgekapselt sind, lange Zeit bestehen können, ohne andere Gefahren, als die, welche jede langwierige Eiterung mit sich bringt, zu involviren. Die die Abscesshöhle auskleidende Schwarte schützt den Körper vor einer Resorption und folgender Allgemeininfection; es ist also nicht die Peritonitis im Sinne der Gewebserkrankung des Bauchfelles, sondern immer Deutsche Zeitschrift f. Chirurgie. XXXIII. Bd. 
nur die Sepsis, die alle weiteren Gefahren mit sich führt, die ihren Eingang in den Körper findet und diesen endlich vernichtet.

Nachdem ich so in fluchtigen Zügen den pathologisch-anatomischen Befund und den Hergang bei Schussverletzungen des MagenDarmtractus skizzirt habe, erlaube ich mir nur noch mit einigen Worten die therapeutische Seite der Frage zu streifen.

Bei richtiger Wurdigung der Thatsache, dass die meisten Schussverletzungen des Magens und Darms von Kotherguss in die Bauchböhle gefolgt sind, die durch Allgemeininfection den Tod herbeiftihrt, kann die Frage, ob wir eine abwartende Therapie einschlagen, oder die Laparotomie machen, kaum im Ernst discutirt werden, da sie sich von selbst beantwortet. Ich habe hier freilich nur die Verhältnisse im Frieden vor Augen, wo wir ja in jeder grösseren Stadt, meist ja wohl auch auf dem Lande die Möglichkeit baben, binnen Kurzem sachverständige Hülfe requiriren zu können. Man könnte höchstens in Discussion treten über die Fragen:

1. Sollen wir in jedem Fall einer perforirenden Verletzung des Abdomens operiren?

2. Wann sollen wir operiren?

Ich glaube die erste Frage bejahen zu dürfen. Die Thierexperimente, sowie die vielfältigen Erfahrungen im Krieg und Frieden haben gelehrt, dass perforirende Schussverletzungen des Abdomens ohne Mitbetheiligung der Eingeweide zu den grössten Seltenheiten gehören. So konnte der vielerfahrene Larrey während seiner langen Dienstzeit nur einen Fall beobachten, wo die durchgehende Kugel unmittelbar keine ernsteren Störungen bewirkt hatte. Später liess sich aber dennoch eine Quetschung des Darmes nachweisen. Wir haben nicht nur das Recht, sondern auch die Pflicht, in jedem Fall einer perforirenden Bauchschussverletzung das Peritoneum zu eröffuen, auch wenn sich einmal herausstellen sollte, dass keine Organverletzung vorlag. Ich möchte den probatorischen Bauchschnitt durchaus nicht als harmlosen Eingriff hinstellen, trotzdem aber meine ich, dass es unter den obwaltenden schwierigen Verhältnissen besser ist, einmal eine Bauchhöhle unnütz aufgeschnitten zu baben, als durch unnöthiges Zaudern in anderen Fällen den sicheren Tod des Patienten herbeizufübren. Die Diagnuse der Organverletzung ist oft schwierig, ja unmöglich, bis auf die Fälle, wo Darm vorgefallen war, sein Inhalt sich nach aussen ergossen hatte, oder eine stärkere Blutung erfolgt war. Es empfiehlt sich daher, nicht lange im Ungewissen zu verharren, sondern durch die Laparotomie Klarbeit zu schaffen und mit dieser den einzigen Weg zur Rettung des Verwundeten zu betreten. 
In der zweiten Frage, betreffend den Zeitpunkt des operativen Eingriffes, ist viel discutirt worden. Ein Theil der Chirurgen stellte sich so, dass erst dann operativ vorgegangen werden sollte, sobald die ersten Zeiehen der Peritonitis ersebienen. Wie machtlos wir aber der einmal ausgebrochenen Sepsis gegentiber sind, zeigen die Berichte der Autoren uiber den Erfolg des Bauchschnittes bei der septischen Peritonitis. Ich erinnere an die Arbeiten von König, Witzel, Steinheil, Miculicz und die vortrefflichen Experimente von Reichel. Wir müssen, wollen wir Erfolg haben, die Operation so schnell wie möglich machen, da durch dieselbe ja hauptsächlich der Ausbruch der allgemeinen Infection verbindert werden soll. Sie soll uns ja die Möglichkeit geben, die Folgen der ins Peritoneum gedrungenen Schädlichkeiten fortzuscbaffen; deshalb also kein Zaudern in der Ausfïhrung der Laparotomie, da jede Verzögerung die Chancen auf einen gïnstigen Erfolg vermindert.

Die perforirenden Verletzungen des Abdomens werden im Krieg und Frieden gewiss noch zahlreiche Opfer fordern; doch hoffen wir, dass mit Erweiterung unserer Kenntnisse auf dem Gebiete der Chirurgie der Bauchhöble sich dem Chirurgen hier noch ein dankbares Feld für seine Kunst bieten wird. 